\title{
On isolated strata of pentagonal Riemann surfaces in the branch locus of moduli spaces
}

Gabriel Bartolini, Antonio F. Costa and Milagros Izquierdo

\section{Linköping University Post Print}

N.B.: When citing this work, cite the original article.

First published as:

Gabriel Bartolini, Antonio F. Costa and Milagros Izquierdo, On isolated strata of pentagonal Riemann surfaces in the branch locus of moduli spaces, 2012, Contemporary Mathematics, (572), 19-24.

Copyright: Providence, RI; American Mathematical Society; 2012 http://www.ams.org/journals/

Postprint available at: Linköping University Electronic Press http://urn.kb.se/resolve?urn=urn:nbn:se:liu:diva-85484 


\title{
On isolated strata of pentagonal Riemann surfaces in the branch locus of moduli spaces
}

\author{
Gabriel Bartolini \\ Matematiska institutionen, \\ Linköpings universitet, \\ 58183 Linköping, Sweden.
}

\author{
Antonio F. Costa* \\ Dept. Matemáticas Fundamentales, \\ Facultad de Ciencias, UNED, \\ 28040 Madrid, Spain.
}

\author{
Milagros Izquierdo ${ }^{\dagger}$ \\ Matematiska institutionen, \\ Linköpings universitet, \\ 58183 Linköping, Sweden.
}

June 20, 2011

\begin{abstract}
The moduli space $\mathcal{M}_{g}$ of compact Riemann surfaces of genus $g$ has orbifold structure, and the set of singular points of such orbifold is the branch locus $\mathcal{B}_{g}$. For $g \not \equiv 3 \bmod 4, g \geq 26, g \neq 37$, there exists isolated strata corresponding to families of pentagonal Riemann surfaces.
\end{abstract}

\section{Introduction}

In this article we study the topology of moduli spaces of Riemann surfaces. The moduli space $\mathcal{M}_{g}$ of compact Riemann surfaces of genus $g$ being the quotient of the Teichmüller space by the discontinuous action of the mapping class group, has the structure of a complex orbifold, whose set of singular points is called the branch locus $\mathcal{B}_{g}$. The branch locus $\mathcal{B}_{g}, g \geq 3$ consists of the Riemann surfaces with symmetry, i. e. Riemann surfaces with nontrivial automorphism group. Our goal is to study of the topology of $\mathcal{B}_{g}$ through its connectedness. The connectedness of moduli spaces of hyperelliptic, $p$-gonal and real Riemann surfaces has been widely studied, for instance by [?], [?], [?], [?], [?].

It is known that $\mathcal{B}_{2}$ is not connected, since R. Kulkarni (see [?] and [?]) showed that the curve $w^{2}=z^{5}-1$ is isolated in $\mathcal{B}_{2}$, i. e. this single surface is a isolated component of $\mathcal{B}_{2}$, furthermore $\mathcal{B}_{2}$ has exactly two connected components (see [?] and [?]). It is also known that the branch loci $\mathcal{B}_{3}, \mathcal{B}_{4}$ and $\mathcal{B}_{7}$ are connected and $\mathcal{B}_{5}, \mathcal{B}_{6}, \mathcal{B}_{8}$ are connected with the exception of isolated points (see [?] and [?]).

In this article we prove that $\mathcal{B}_{g}$ is disconnected for $g \not \equiv 3 \bmod 4, g \geq 26$; more concretely we find equisymmetric isolated strata induced by order 5 automorphisms of Riemann surfaces of genera $g \not \equiv 3 \bmod 4$. In [?] it is proved that $\mathcal{B}_{g}$ is disconnected for $g \geq 65$.

\footnotetext{
*Partially supported by MTM2008-00250

${ }^{\dagger}$ Partially supported by the Swedish Research Council (VR)
} 
We wish to thank Rubén Hidalgo for a revision of the article and several suggestions.

\section{Riemann surfaces and Fuchsian groups}

Let $X$ be a Riemann surface and assume that $\operatorname{Aut}(X) \neq\{1\}$. Hence $X / \operatorname{Aut}(X)$ is an orbifold and there is a Fuchsian group $\Gamma \leq \operatorname{Aut}(\mathcal{D})$, such that $\pi_{1}(X) \triangleleft \Gamma$ :

$$
\mathcal{D} \rightarrow X=\mathcal{D} / \pi_{1}(X) \rightarrow X / A u t(X)=\mathcal{D} / \Gamma
$$

where $\mathcal{D}=\{z \in \mathbb{C}:\|z\|<1\}$.

If the Fuchsian group $\Gamma$ is isomorphic to an abstract group with canonical presentation

$$
\left\langle a_{1}, b_{1}, \ldots, a_{g}, b_{g}, x_{1} \ldots x_{k} \mid x_{1}^{m_{1}}=\cdots=x_{k}^{m_{k}}=\prod_{i=1}^{k} x_{i} \prod_{i=1}^{g}\left[a_{i}, b_{i}\right]=1\right\rangle,
$$

we say that $\Gamma$ has signature

$$
s(\Gamma)=\left(g ; m_{1}, \ldots, m_{k}\right) .
$$

The generators in presentation (??) will be called canonical generators.

Let $X$ be a Riemann surface uniformized by a surface Fuchsian group $\Gamma_{g}$, i.e. a group with signature $(g ;-)$. A finite group $G$ is a group of automorphisms of $X$, i.e. there is a holomorphic action $a$ of $G$ on $X$, if and only if there is a Fuchsian group $\Delta$ and an epimorphism $\theta_{a}: \Delta \rightarrow G$ such that $\operatorname{ker} \theta_{a}=\Gamma_{g}$. The epimorphism $\theta_{a}$ is the monodromy of the covering $f_{a}: X \rightarrow X / G=\mathcal{D} / \Delta$.

The relationship between the signatures of a Fuchsian group and subgroups is given in the following theorem of Singerman:

Theorem 1 ([?]) Let $\Gamma$ be a Fuchsian group with signature (??) and canonical presentation (??). Then $\Gamma$ contains a subgroup $\Gamma^{\prime}$ of index $N$ with signature

$$
s\left(\Gamma^{\prime}\right)=\left(h ; m_{11}^{\prime}, m_{12}^{\prime}, \ldots, m_{1 s_{1}}^{\prime}, \ldots, m_{k 1}^{\prime}, \ldots, m_{k s_{k}}^{\prime}\right) .
$$

if and only if there exists a transitive permutation representation $\theta: \Gamma \rightarrow \Sigma_{N}$ satisfying the following conditions:

1. The permutation $\theta\left(x_{i}\right)$ has precisely $s_{i}$ cycles of lengths less than $m_{i}$, the lengths of these cycles being $m_{i} / m_{i 1}^{\prime}, \ldots, m_{i} / m_{i s_{i}}^{\prime}$.

2. The Riemann-Hurwitz formula

$$
\mu\left(\Gamma^{\prime}\right) / \mu(\Gamma)=N .
$$

where $\mu(\Gamma), \mu\left(\Gamma^{\prime}\right)$ are the hyperbolic areas of the surfaces $\mathcal{D} / \Gamma, \mathcal{D} / \Gamma^{\prime}$.

For $\mathcal{G}$, an abstract group isomorphic to all the Fuchsian groups of signature $s=\left(h ; m_{1}, \ldots, m_{k}\right)$, the Teichmüller space of Fuchsian groups of signature $s$ is:

$$
\{\rho: \mathcal{G} \rightarrow P S L(2, \mathbb{R}): s(\rho(\mathcal{G}))=s\} / \text { conjugation in } P S L(2, \mathbb{R})=T_{s} .
$$


The Teichmüller space $T_{s}$ is a simply-connected complex manifold of dimension $3 g-3+k$. The modular group, $M(\Gamma)$, of $\Gamma$, acts on $T(\Gamma)$ as $[\rho] \rightarrow[\rho \circ \alpha]$ where $\alpha \in M(\Gamma)$. The moduli space of $\Gamma$ is the quotient space $\mathcal{M}(\Gamma)=T(\Gamma) / M(\Gamma)$, then $\mathcal{M}(\Gamma)$ is a complex orbifold and its singular locus is $\mathcal{B}(\Gamma)$, called the branch locus of $\mathcal{M}(\Gamma)$. If $\Gamma_{g}$ is a surface Fuchsian group, we denote $\mathcal{M}_{g}=T_{g} / M_{g}$ and the branch locus by $\mathcal{B}_{g}$. The branch locus $\mathcal{B}_{g}$ consists of surfaces with non-trivial symmetries for $g>2$.

If $X / \operatorname{Aut}(X)=\mathcal{D} / \Gamma$ and genus $(X)=g$, then there is a natural inclusion $i: T_{s} \rightarrow T_{g}:[\rho] \rightarrow\left[\rho^{\prime}\right]$, where

$$
\rho: \mathcal{G} \rightarrow P S L(2, \mathbb{R}), \pi_{1}(X) \subset \mathcal{G}, \rho^{\prime}=\left.\rho\right|_{\pi_{1}(X)}: \pi_{1}(X) \rightarrow P S L(2, \mathbb{R}) .
$$

If we have $\pi_{1}(X) \triangleleft \mathcal{G}$, then there is a topological action of a finite group $G=\mathcal{G} / \pi_{1}(X)$ on surfaces of genus $g$ given by the inclusion $a: \pi_{1}(X) \rightarrow \mathcal{G}$. This inclusion $a: \pi_{1}(X) \rightarrow \mathcal{G}$ produces $i_{a}\left(T_{s}\right) \subset T_{g}$.

The image of $i_{a}\left(T_{s}\right)$ by $T_{g} \rightarrow \mathcal{M}_{g}$ is $\overline{\mathcal{M}}^{G, a}$, where $\overline{\mathcal{M}}^{G, a}$ is the set of Riemann surfaces with automorphisms group containing a subgroup acting in a topologically equivalent way to the action of $G$ on $X$ given by the inclusion $a$, see [?], the subset $\mathcal{M}^{G, a} \subset \overline{\mathcal{M}}^{G, a}$ is formed by the surfaces whose full group of automorphisms acts in the topologically way given by $a$. The branch locus, $\mathcal{B}_{g}$, of the covering $T_{g} \rightarrow \mathcal{M}_{g}$ can be described as the union $\mathcal{B}_{g}=\bigcup_{G \neq\{1\}} \overline{\mathcal{M}}^{G, a}$, where $\left\{\mathcal{M}^{G, a}\right\}$ is the equisymmetric stratification of the branch locus [?]:

Theorem 2 ([?]) Let $\mathcal{M}_{g}$ be the moduli space of Riemann surfaces of genus $g, G$ a finite subgroup of the corresponding modular group $M_{g}$. Then:

(1) $\overline{\mathcal{M}}_{g}^{G, a}$ is a closed, irreducible algebraic subvariety of $\mathcal{M}_{g}$.

(2) $\mathcal{M}_{g}^{G, a}$, if it is non-empty, is a smooth, connected, locally closed algebraic subvariety of $\mathcal{M}_{g}$, Zariski dense in $\overline{\mathcal{M}}_{g}^{G, a}$.

There are finitely many strata $\mathcal{M}_{g}^{G, a}$.

An isolated stratum $\mathcal{M}^{G, a}$ in the above stratification is a stratum that satisfies $\overline{\mathcal{M}}^{G, a} \cap \overline{\mathcal{M}}^{H, b}=\varnothing$, for every group $H$ and action $b$ on surfaces of genus $g$. Thus $\overline{\mathcal{M}}^{G, a}=\mathcal{M}^{G, a}$

Since each non-trivial group $G$ contains subgroups of prime order, we have the following remark:

Remark 3 ([?])

$$
\mathcal{B}_{g}=\bigcup_{p \text { prime }} \overline{\mathcal{M}}^{C_{p}, a}
$$

where $\overline{\mathcal{M}}^{C_{p}, a}$ is the set of Riemann surfaces of genus $g$ with an automorphism group containing $C_{p}$, the cyclic group of order $p$, acting on surfaces of genus $g$ in the topological way given by $a$.

\section{Disconnectedness by pentagonal Riemann sur- faces}

By the Castelnuovo-Severi inequality [?], the $p$-gonal morphism of an ellipticor $p$ - gonal Riemann surface $X_{g}$ of genus $g$ is unique if $g \geq 2 h p+(p-1)^{2}+1$, where $h \in\{0,1\}$ is the genus of the quotient surface. 
Let $X_{g}, g \geq 10 h+17$, be an (elliptic-) pentagonal surface, such that $X_{g} \in \overline{\mathcal{M}}_{g}^{C_{5}, a}$ for some action $a$, let $\langle\alpha\rangle$ be the group of (elliptic-) pentagonal automorphisms of $X_{g}$. Consider an automorphism $b \in A u t(X) \backslash\langle\alpha\rangle$, by the Castelnuovo-Severi inequality, $b$ induces an automorphism $\hat{b}$ of order $p$ on the Riemann surface $X_{g} /\langle a\rangle=Y_{h}$, of genus $h$, according to the following diagram:

$$
\begin{array}{cccc}
X_{g}=\mathcal{D} / \Gamma_{g} & \stackrel{b}{\rightarrow} & X_{g}=\mathcal{D} / \Gamma_{g} \\
& f_{a} \downarrow & & \downarrow f_{a} \\
X_{g} /\langle\alpha\rangle=Y_{h}\left(P_{1}, \ldots, P_{k}\right) & \stackrel{\hat{b}}{\rightarrow} & X_{g} /\langle\alpha\rangle= & Y_{h}\left(P_{1}, \ldots, P_{k}\right)
\end{array}
$$

where $\Gamma_{g}$ is a surface Fuchsian group and $f_{a}: X_{g}=\mathcal{D} / \Gamma_{g} \rightarrow X_{g} /\langle\alpha\rangle$ is the morphism induced by the group of automorphisms $\langle\alpha\rangle$ with action $a$. $S=\left\{P_{1}, \ldots, P_{k}\right\}$ is the branch set in $Y_{h}$ of the morphism $f_{a}$ with monodromy $\theta_{a}: \Delta(h ; 5, . k ., 5) \rightarrow C_{5}$ defined by $\theta_{a}\left(x_{i}\right)=\alpha^{t_{i}}$, where $t_{i} \in\{1,2,3,4\}$ for $1 \leq i \leq k$. Let $n_{j}$ denote the number of times that the exponents $j$ occurs among $t_{1}, \ldots, t_{k}$, for $1 \leq j \leq 4$. Then $n_{1}+n_{2}+n_{3}+n_{4}=k$ and $1 n_{1}+2 n_{2}+3 n_{3}+4 n_{4} \equiv 0 \bmod 5$.

Now, $\hat{b}$ induces a permutation on $S$ that either takes singular points with monodromy $\alpha^{j}$ to points with monodromy $\alpha^{5-j}$, takes points with monodromy $\alpha^{j}$ to points with monodromy $\alpha^{2 j}$, or it acts on each subset formed by points in $S$ with same monodromy $\alpha^{t_{j}}$. Therefore the following conditions force $\hat{b}$ to be the identity on $Y_{h}$ :

1. $\left|n_{1}-n_{4}\right|+\left|n_{2}-n_{3}\right| \geq 3+h$

2. $\left|n_{1}-n_{j}\right| \geq 3+h$, for some $n_{j}$ such that $2 \leq j \leq 4$ and

3.let $\widehat{n_{j}} \in\{1,2,3,4\}$, such that $\widehat{n_{j}} \equiv n_{j} \bmod p$, then $\sum_{j=1}^{4} \widehat{n_{j}} \geq 3+h$.

Theorem 4 Assume $g \geq 18$ is even, then there exist isolated strata formed by pentagonal surfaces.

Proof. We will construct monodromies $\theta: \Delta(0 ; 5, . k ., 5) \rightarrow C_{5}$, where $k=\frac{g}{2}+2$ by the Riemann-Hurwitz formula, such that the conditions (??) above are satisfied. Assume $\theta\left(x_{i}\right)=\alpha^{t_{i}}, i=1, \ldots, k$. Let $n_{j}=\mid\left\{t_{i}=j ; i=\right.$ $1, \ldots, k\} \mid$, then we will define the epimorphism $\theta$ by the generating vector $\left(n_{1} \alpha, n_{2} \alpha^{2}, n_{3} \alpha^{3}, n_{4} \alpha^{4}\right)$, where $n_{j} \alpha^{j}$ means that $\alpha^{j}$ is the monodromy of $n_{j}$ different singular points $P_{i}$.

\begin{tabular}{c|c|cccc}
$g \bmod 5$ & $k \bmod 5$ & $n_{1}$ & $n_{2}$ & $n_{3}$ & $n_{4}$ \\
\hline$g \equiv 0 \bmod 5$ & $k \equiv 2 \bmod 5$ & $(k-13)$ & 5 & 1 & 7 \\
\hline$g \equiv 1 \bmod 5$ & $k \equiv 0 \bmod 5$ & $(k-7)$ & 5 & 1 & 1 \\
\hline$g \equiv 2 \bmod 5$ & $k \equiv 3 \bmod 5$ & $(k-9)$ & 1 & 3 & 5 \\
\hline$g \equiv 3 \bmod 5$ & $k \equiv 1 \bmod 5$ & $(k-7)$ & 1 & 5 & 1 \\
\hline$g \equiv 4 \bmod 5$ & $k \equiv 4 \bmod 5$ & $(k-9)$ & 5 & 1 & 3
\end{tabular}

We see that the given epimorphisms satisfy the conditions (??) except for $g=20, k=12$. However, in this case, $g=20, k=12$, let the epimorphism $\theta: \Delta(0 ; 5,12,5) \rightarrow C_{5}$ be defined by the generating vector $\left(\alpha, 7 \alpha^{2}, \alpha^{3}, 3 \alpha^{4}\right)$. $\theta$ clearly satisfies the conditions ?? above. 
Remark 5 The complex dimension of the isolated strata given in the proof of theorem ?? is $0 \times 3-3+k=g / 2+2-3=g / 2-1$.

Remark 6 There are several isolated strata of dimension $g / 2-1$ in $\mathcal{B}_{g}$ for even genera $g \geq 22$. For instance consider $g \equiv 3 \bmod 5$. The monodromy $\theta^{\prime}$ defined by the generating vector $\left((k-9) \alpha, 5 \alpha^{2}, 3 \alpha^{3}, 5 \alpha^{4}\right)$ induces an isolated stratum different from the one given in the proof of Theorem ?? since the actions determined by $\theta$ and $\theta^{\prime}$ are not topologically equivalent, see [?].

Theorem 7 Assume $g \geq 29, g \equiv 1 \bmod 4, g \neq 37$, then there exists isolated strata formed by elliptic-pentagonal surfaces.

Proof. Similarly to the proof of Theorem ??, using the conditions above (??), we will construct epimorphisms $\theta: \Delta(1 ; 5, . k ., 5) \rightarrow C_{5}$, where $k=$ $\frac{g-1}{2}$ by the Riemann-Hurwitz formula. Assume $\theta\left(x_{i}\right)=\alpha^{t_{i}}, i=1, \ldots, k$. Let $n_{j}=\left|\left\{t_{i}=j ; i=1, \ldots, k\right\}\right|$, the epimorphism $\theta$ will be defined by the generating vector $\left(n_{1} \alpha, n_{2} \alpha^{2}, n_{3} \alpha^{3}, n_{4} \alpha^{4}\right)$, where $n_{j} \alpha^{j}$ means that $\alpha^{j}$ appears as the monodromy of $n_{j}$ different singular points $P_{i}$.

\begin{tabular}{c|c|cccc}
\hline$g \bmod 5$ & $k \bmod 5$ & $n_{1}$ & $n_{2}$ & $n_{3}$ & $n_{4}$ \\
\hline$g \equiv 0 \bmod 5$ & $k \equiv 2 \bmod 5$ & $(k-13)$ & 5 & 1 & 7 \\
\hline$g \equiv 1 \bmod 5$ & $k \equiv 0 \bmod 5$ & $(k-7)$ & 5 & 1 & 1 \\
\hline$g \equiv 2 \bmod 5$ & $k \equiv 3 \bmod 5$ & $(k-19)$ & 11 & 3 & 5 \\
\hline$g \equiv 3 \bmod 5$ & $k \equiv 1 \bmod 5$ & $(k-7)$ & 1 & 5 & 1 \\
\hline$g \equiv 4 \bmod 5$ & $k \equiv 4 \bmod 5$ & $(k-11)$ & 1 & 5 & 5
\end{tabular}

We see that the given epimorphisms satisfy the conditions set except for $g=37, k=18$.

Remark 8 The complex dimension of the isolated strata given in the proof of theorem ?? is $1 \times 3-3+k=(g-1) / 2$.

Remark 9 Let $g \equiv 3 \bmod 4$. Then there is no isolated stratum in $\mathcal{B}_{g}$ of dimension $(g-1) / 2$. Such a stratum will consist of elliptic-pentagonal surfaces given by epimorphisms $\theta: \Delta(1 ; 5, . k ., 5) \rightarrow C_{5}$, where $k=(g-$ 1) $/ 2 \equiv 1 \bmod 2$. Now such epimorphisms cannot satisfy the third condition in (??).

\section{References}

[A] Accola, R. D. M. (1984) On cyclic trigonal Riemann surfaces. I. Trans. Amer. Math. Soc. 283 no. 2, 423-449.

[BCIP] Bartolini, G., Costa, A.F., Izquierdo, M., Porto, A.M., (2010) On the connectedness of the branch locus of the moduli space of Riemann surfaces, RACSAM Rev. R. Acad. Cienc. Exactas Fís. Nat. Ser. A Mat. 104 no.1 81-86.

[BI] Bartolini, G., Izquierdo, M. (2010) On the connectedness of branch loci of moduli spaces of Riemann surfaces of low genus. To appear in Proc. Amer. Math. Soc. 
[Bo] Bolza, O. (1888) On binary sextics with linear transformations between themselves, Amer. J. Math. 10, 47-70.

[B] Broughton, S. A. (1990) The equisymmetric stratification of the moduli space and the Krull dimension of mapping class groups. Topology Appl. 37 $101-113$.

[BCI] Bujalance, E.; Costa, A. F.; Izquierdo, M. (1998) A note on isolated points in the branch locus of the moduli space of compact Riemann surfaces. Ann. Acad. Sci. Fenn. Math. 23 no. 1, 25-32.

[BSS] Buser, P., Seppälä, M. , Silhol, R.(1995) Triangulations and moduli spaces of Riemann surfaces with group actions. Manuscripta Math. 88 209-224.

[CI1] Costa, A. F., Izquierdo, M. (2002) On the connectedness of the locus of real Riemann surfaces. Ann. Acad. Sci. Fenn. Math. 27 341-356.

[CI2] Costa, A. F., Izquierdo, M. (2010) On the connectedness of the branch locus of the moduli space of Riemann surfaces of genus 4. Glasg. Math. J. 52 (2010), no. 2, 401-408.

[CI3] Costa, A. F., Izquierdo, M. (2009) On the existence of connected components of dimension one in the branch loci of moduli spaces of Riemann surfaces. Preprint.

[CI4] Costa, A. F., Izquierdo, M. (2010) Equisymmetric strata of the singular locus of the moduli space of Riemann surfaces of genus 4. LMS Lect. Note Series 368 130-148.

[CI5] Costa, A. F., Izquierdo, M. (2011) On the connectivity of branch loci of moduli spaces. Preprint.

[Co] Cornalba, M. (1987) On the locus of curves with automorphisms. Annali di Matematica Pura e Applicata (4) 149, 135-151.

[G] González-Díez, G. (1995). On prime Galois covering of the Riemann sphere. Ann. Mat. Pure Appl. 168 1-15

[H] Harvey, W. (1971) On branch loci in Teichmüller space. Trans. Amer. Math. Soc. 153 387-399.

[K] Kulkarni, R. S. (1991) Isolated points in the branch locus of the moduli space of compact Riemann surfaces. Ann. Acad. Sci. Fen. Ser. A I MAth. $1671-81$.

[Se] Seppälä, M. (1990) Real algebraic curves in the moduli space of complex curves. Comp. Math., 74 259-283.

[Si1] Singerman, D. (1970) Subgroups of Fuchsian groups and finite permutation groups Bull. London Math. Soc. 2 319-323.

[Si2] Singerman, D. (1972) Finitely maximal Fuchsian groups. J. London Math. Soc. 6 29-38. 